\title{
Progressive multiple cranial neuropathies presenting as a delayed complication of radiotherapy in infancy
}

\author{
Hardev S. Pall, ${ }^{1}$ Simon Nightingale, ${ }^{1}$ Christopher G. Clough ${ }^{1}$ and \\ David Spooner ${ }^{2}$
}

Departments of ${ }^{1}$ Neurology and ${ }^{2}$ Radiotherapy, Queen Elizabeth Hospital, Queen Elizabeth Medical Centre, Edgbaston, Birmingham B15 2TH, UK.

\begin{abstract}
Summary: A 38 year old woman who had undergone irradiation during infancy for a left facial cutaneous arteriovenous malformation sequentially developed complete palsies of the ipsilateral VII, V, XI, IX, X, XII and VI cranial nerves.

Apart from optic and olfactory nerve damage there are few reports of radiotherapy causing cranial nerve injury. We link the unusually extensive and progressive neural damage and the prolonged latency to the patient's age at time of irradiation.
\end{abstract}

\section{Introduction}

Damage to the peripheral nervous system including the cranial nerves is fortunately an uncommon complication of radiotherapy treatment. In this paper we describe an exceptional case of progressive multiple cranial neuropathies presenting as a delayed complication of radiotherapy in infancy.

\section{Case report}

In 1983 a 38 year old woman presented with a 4year history of progressive left facial and tongue dysaesthesia followed sequentially by weakness of left shoulder shrugging, liquid regurgitation into the left nostril, difficulty with speech and swallowing and more recently, diplopia.

At the age of 7 months she had received radiotherapy for a left facial cutaneous arteriovenous malformation. The therapy comprised irradiation with $85 \mathrm{kV}$ photons resulting in a total skin radiation dose of 1000 roentgen $(1130 \mathrm{rad})$ in four treatments over 20 weeks (Approximate Nominal Standard Dose $=420$ ret). This was directed at a single field of $10 \mathrm{~cm}$ in diameter which would cover most of an infant's face in profile. In addition, between 1949 and 1955 she had 23 applications of radioactive thorium $\left({ }^{228} \mathrm{Th}\right)$ paint to skin overlying the malformation. In 1954 at the age of 9 years she

developed left lower motor neurone facial weakness without any associated ear, nose or throat problems.

Examination in 1983 showed a complete left VII nerve palsy including a dry eye (Schirmer's test), loss of the stapedius reflex and loss of taste over the anterior two thirds of the left side of the tongue. She also had pareses of the left V, VI, X, $\mathrm{XI}$, and XII nerves and anaesthesia in the left V and IX nerve distributions. She still had an arteriovenous malformation over the left face, neck and scalp. There were no signs of involvement of the central nervous system (CNS).

Investigations including blood count, erythrocyte sedimentation rate, treponemal serology, serum antinuclear factor, Kveim test, cerebrospinal fluid examination, plain skull X-ray, computerized tomography of the head with intravenous and intracisternal contrast media, four vessel cranial angiography and magnetic resonance imaging in 1985 were normal. Examination of her ears, nose and throat did not provide any additional information. When reviewed in 1987 no further change in the symptoms or clinical signs had occurred.

\section{Discussion}

Late-delayed post-radiotherapy changes are considered the likely explanation for this patient's cranial nerve palsies. The failure of extensive

(C) The Fellowship of Postgraduate Medicine 1988 
investigation and prolonged follow-up supports this view. Adverse neurological reactions to cranial irradiation are usually confined to the CNS. ${ }^{1}$ Damage to the olfactory and optic nerves (which form part of the CNS) following radiotherapy is well described, ${ }^{1}$ but instances of damage to other cranial nerves are poorly documented. The few studies reporting cranial nerve damage have described adults who received large dose, radical, mega-voltage radiotherapy (Nominal Standard Dose usually greater than 1800 ret). The series described by Berger \& Bataini ${ }^{2}$ consists of patients who sustained radiotherapy damage to cranial nerves with an extracranial course. The patient that we have described had a VI nerve palsy, demonstrating that damage was not confined to extracranial structures, although there were no signs of central nervous system involvement. We have been unable to find a previous report of such progressive and extensive post-radiotherapy cranial nerve damage that firstly, continued for three decades; secondly occurred after such a modest dose of radiation and thirdly affected the intracranial course of cranial nerves without damaging central nervous structures.

The mean thickness of the female infant skull at one year is $0.3 \mathrm{~cm}$ compared with $0.9 \mathrm{~cm}$ at age 25 years $^{3}$ and infant bones have less calcium than adult bones. ${ }^{4}$ The penetration of radiation through bone is markedly increased by reduction in either its thickness or its calcium content. For both these reasons the radioprotection offered by the skull and facial bones in our patient's case may have been insufficient to protect the cranial nerves but sufficient to protect the even deeper central nervous structures.

The decreased 'radioprotection' by the infant skull may be partly responsible for neural toxicity occurring at a total radiation dose that is well within 'safe limits' for adult and late childhood irradiation. Berger \& Bataini ${ }^{2}$ reported 1800 ret to be the lowest dose of radiation which in their series caused cranial nerve damage. This is much higher than the $\mathbf{4 7 0}$ ret that our patient received. However, single radiation doses as low as 5 rad may damage the fetal nervous system. ${ }^{5}$ In the same way, it is possible that the incompletely developed nervous system of the infant is more susceptible than the adult nervous system to the effects of radiation.

Thorium $\left({ }^{228} \mathrm{Th}\right)$ paint has a half-life of 1.91 years and almost entirely emits alpha particles which have a very limited capacity to penetrate tissues. The thorium paint is therefore unlikely to have contributed significantly to the patient's postradiotherapy neuropathies.

The long latency between radiotherapy and cranial nerve damage in our patient (over 400 months) is exceptional. Berger and Bataini report a patient who developed cranial neuropathies after a latency of 172 months. ${ }^{2}$ In Sheline's series of 80 cases of late-delayed postradiotherapy CNS necrosis, the longest latency was 90 months, ${ }^{6}$ but damage to peripheral nerves may follow latencies of several decades. ${ }^{7}$ Since cranial nerves (other than the optic and olfactory nerves) are more closely analogous to peripheral nerves than CNS neurones, the characteristics of the complications of radiotherapy in peripheral and cranial nerves may be similar. In their studies Berger and Bataini found an inverse linear relationship between the Nominal Standard Dose and the delay until the onset of cranial nerve symptoms. ${ }^{2}$ It appears that low doses result in prolonged latencies to the onset of cranial nerve damage, although such damage is in fact less likely with lower doses.

The cranial nerves with a superficial path absorb a greater dose of radiation and may be expected to express clinical damage after shorter latencies. A crude estimate of the minimum distance between skin and cranial nerves was obtained by cadaver dissection and direct measurement. Figure 1 shows that except for cranial nerve VII, there is a linear relationship between latency and minimum distance of the nerve from skin, providing confirmation that the observed clinical damage is a consequence of radiotherapy. The markedly shorter latency to damage of the VII nerve may be due to the extensive superficial course of the facial nerve along the lateral aspect of the face.

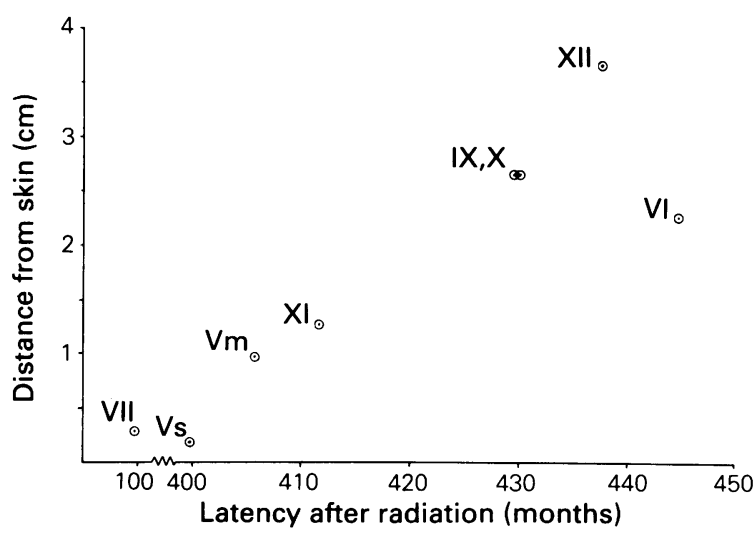

Figure 1 The relationship of the distance of the cranial nerve from the skin to the latency of onset of the neuropathy following radiation. Spearman's rankorder correlation coefficient is $0.83, P=0.02$. Vs and $\mathrm{Vm}$ are the sensory and motor components respectively of the trigeminal nerve. 
Whereas high dose irradiation reproducibly causes tissue damage, the doses used routinely in radiotherapy only infrequently result in neurological complications. However, in some instances exemplified by our patient, extensive neural damage may result even after several decades. Furthermore, the clinical pattern of neural damage following

\section{References}

1. Thomas, P.K. \& Holdorf,, B. Neuropathy due to physical agents. In: Dyck, P.J., Thomas, P.K., Lambert, E.H. \& Bunge, R. (eds) Peripheral Neuropathy, Vol 2. W.B. Saunders, Philadelphia, 1984, p 1948.

2. Berger, P.S. \& Bataini, J.P. Radiation induced cranial nerve palsy. Cancer 1977, 40: 152-155.

3. Lusted, L.B. \& Keats, T.E. Atlas of Roentgenographic Measurement, 3rd Ed. Year Bk. Med., Chicago, 1972, p 37.

4. Report of the task group on reference man. International Commission on Radiological Protection, No 23. Pergamon, Oxford, 1975, p 73. irradiation in infancy may be quite different from that seen with irradiation in later childhood and adult life.

\section{Acknowledgement}

We are grateful to Dr Michael Small for permission to report his patient.

5. Rugh, R. Major radiobiological concepts and effects of ionising radiation on the embryo and fetus. In: Haley, T.J. and Snider, R.S. (eds) Response of the Nervous System to Ionising Radiation. Academic Press, New York, 1962, pp 3-26.

6. Sheline, G.E., Wara, W.M. \& Smith, V. Therapeutic irradiation and brain injury. Int J Radiat Oncol Biol Phys 1980, 6: 1215-1228.

7. Kori, S.H., Foley, K.M. \& Posner, J.B. Brachial plexus lesions in patients with cancer: 100 cases. Neurology 1981, 31: 45-50. 